\title{
Segurança e Eficácia do Acesso Radial na Realização de Procedimentos Coronários Diagnósticos e Terapêuticos em Mulheres
}

\author{
Pedro Beraldo de Andrade ${ }^{1,2}$, Marden André Tebet ${ }^{1,2}$, Mônica Vieira Athanazio de Andrade', \\ Luiz Alberto Mattos ${ }^{1,3}$, André Labrunie ${ }^{1,2}$
}

\section{RESUMO}

Introdução: O sexo feminino representa um preditor independente para ocorrência de complicações vasculares em pacientes submetidos a procedimentos coronários invasivos. Ao reduzir o risco de sangramento relacionado ao sítio de punção, a utilização do acesso radial em mulheres seria particularmente benéfica na redução de eventos adversos. Método: Pacientes consecutivas do sexo feminino submetidas a procedimentos coronários diagnósticos e terapêuticos pela via radial foram incluídas em um registro prospectivo. Foram avaliados o sucesso da técnica, a duração do procedimento e da fluoroscopia, as complicações vasculares e os eventos cardiovasculares adversos graves periprocedimento. Realizou-se adicionalmente uma análise comparativa entre mulheres com $<65$ anos e com $>65$ anos de idade, a fim de determinar a aplicabilidade da técnica entre as idosas. Resultados: Entre maio de 2008 e setembro de 2009, 988 mulheres submeteram-se a procedimentos coronários invasivos pelo acesso radial. A média de idade foi de 62,5 anos, sendo $32,4 \%$ portadoras de diabetes melito. A taxa de sucesso foi de $97,1 \%$. Houve baixa incidência de complicações, incluindo $2,8 \%$ de oclusão assintomática da artéria radial, 2,4\% de hematomas e $0,2 \%$ de sangramento grave. Exceto por maior duração da fluoroscopia durante a realização de angioplastias, as mulheres do grupo com $\geq 65$ anos exibiram similares taxas de sucesso e complicações quando comparadas àquelas do grupo com < 65 anos. Conclusões: $O$ uso do acesso radial na realização de procedimentos coronários em mulheres exibiu elevada taxa de sucesso, reduzido número de eventos cardiovasculares adversos e ocorrência virtualmente nula de sangramento grave relacionado ao sítio de punção.

DESCRITORES: Doenças vasculares/complicações. Cateterismo cardíaco. Artéria radial. Angioplastia transluminal percutânea coronária. Síndrome coronariana aguda. Fatores sexuais. Mulheres.

\author{
ABSTRACT \\ Safety and Efficacy of the Transradial Approach \\ in Diagnostic and Therapeutic Coronary \\ Procedures in Women
}

Background: Female gender is an independent predictor of vascular complications after invasive coronary procedures. While reducing the risk of bleeding related to the puncture site, radial access in women might be particularly beneficial in reducing adverse events. Method: Consecutive female patients undergoing diagnostic and therapeutic coronary procedures using radial access were enrolled in a prospective registry. The success of the technique, the procedure and fluoroscopy times, vascular complications and severe periprocedural cardiovascular adverse events were analyzed. In addition, a comparative analysis between women $<65$ and $\geq 65$ years old was carried out to determine the applicability of the technique among elderly patients. Results: Between May 2008 and September 2009, 988 women underwent invasive coronary procedures using the radial access. Mean age was 62.5 years, and $32.4 \%$ were diabetics. The success rate was $97.1 \%$. There was a low incidence of complications, including asymptomatic occlusion of the radial artery $(2.8 \%)$, hematomas $(2.4 \%)$, and severe bleeding $(0.2 \%)$. Except for a longer duration of fluoroscopy while performing angioplasties, women $\geq 65$ years showed similar success rates compared to those $<65$ years. Conclusions: The use of radial access for coronary procedures in women showed a high success rate, reduced number of adverse cardiovascular events, and virtually no severe bleeding related the puncture site.

DESCRIPTORS: Vascular diseases/complications. Heart catheterization. Radial artery. Angioplasty, transluminal percutaneous coronary. Acute coronary syndrome. Sex factors. Women.

1 Irmandade da Santa Casa de Misericórdia de Marília - Marília, SP, Brasil.

${ }^{2}$ Hospital do Coração de Londrina - Londrina, PR, Brasil.

3 Instituto Dante Pazzanese de Cardiologia - São Paulo, SP, Brasil.

Correspondência: Pedro Beraldo de Andrade. Irmandade da Santa

Casa de Misericórdia de Marília. Avenida Vicente Ferreira, 828 -

Marília, SP, Brasil - CEP 17515-900

E-mail: pedroberaldo@gmail.com

Recebido em: 27/9/2009 • Aceito em: 31/10/2009 
l: mportantes avanços na farmacoterapia antitrombótica adjunta e no desenvolvimento de novos dispositivos possibilitaram a redução de complicações relacionadas a procedimentos coronários invasivos na última década ${ }^{1,2}$. No entanto, o risco de complicações vasculares, notadamente de sangramento, entre as mulheres permanece elevado quando comparado aos homens, tornando o sexo feminino um dos principais preditores para a ocorrência desse desfecho adver$\mathrm{so}^{3,4}$. Com o acúmulo de publicações, encontra-se hoje estabelecida a forte associação entre sangramento grave e mortalidade em pacientes submetidos a intervenção coronária percutânea e com diagnóstico de síndrome coronária aguda 5 .

Nesse contexto, a utilização do acesso radial em mulheres torna-se particularmente vantajosa. Comparado ao acesso femoral em meta-análise de ensaios randômicos, associou-se a redução de complicações relacionadas ao sítio de punção, menor tempo de hospitalização e custo hospitalar, com taxa similar de eventos cardiovasculares adversos graves ${ }^{6}$. Em nova e recente revisão sistemática, envolvendo maior número de pacientes, o acesso radial promoveu redução significativa da taxa de sangramento grave, com tendência a menor ocorrência de morte, infarto agudo do miocárdio e acidente vascular encefálico ${ }^{7}$.

No entanto, exibindo menor área de superfície corpórea e menor diâmetro da artéria radial ${ }^{8}$, pacientes do sexo feminino frequentemente são preteridas quanto à escolha dessa via, dado o receio de falha na tentativa de punção ou ocorrência de espasmo. O presente estudo tem por objetivo avaliar a segurança e a eficácia do acesso radial na realização de procedimentos coronários diagnósticos e terapêuticos em mulheres, na prática contemporânea, representativa do mundo real, em centro em que a utilização dessa via é priorizada.

\section{MÉTODO}

Pacientes consecutivas do sexo feminino submetidas a procedimentos coronários diagnósticos e terapêuticos pelo acesso radial foram incluídas em um registro prospectivo de eficácia e segurança. A eficácia da técnica radial foi avaliada por meio da taxa de sucesso do procedimento, definido como realização de cinecoronariografia com adequada opacificação coronária e intervenção coronária percutânea obtendose lesão residual inferior a $30 \%$, sem necessidade de mudança da via de acesso (crossover). Os tempos de procedimento e fluoroscopia foram obtidos a partir do início da punção arterial até a retirada do introdutor. A segurança foi avaliada por meio da ocorrência de complicações vasculares relacionadas ao sítio de punção e eventos cardiovasculares adversos graves periprocedimento. Por fim, realizou-se uma análise comparativa entre pacientes com $<65$ anos e com $\geq 65$ anos, a fim de determinar a aplicabilidade da técnica radial em mulheres idosas.
Classificou-se como evento cardiovascular adverso grave a ocorrência de morte, infarto agudo do miocárdio, acidente vascular encefálico, cirurgia de revascularização miocárdica de emergência ou sangramento grave. Este foi definido como hemorragia intracraniana, intraocular, retroperitoneal, queda de hemoglobina superior a $3 \mathrm{~g} / \mathrm{dl}$ ou necessidade de transfusão sanguínea. Os hematomas foram graduados de acordo com a classificação do estudo Early Discharge after Transradial Stenting of Coronary Arteries (EASY) ${ }^{9}$ : tipo I, $\leq 5 \mathrm{~cm}$ de diâmetro; tipo II, $\leq 10 \mathrm{~cm}$ de diâmetro; tipo III, > $10 \mathrm{~cm}$, sem atingir o cotovelo; tipo IV, hematoma estendendo-se além do cotovelo; e tipo $\mathrm{V}$, qualquer hematoma com injúria isquêmica à mão. Complicações relacionadas ao sítio de punção além de hematomas incluíram fístula arteriovenosa, pseudoaneurisma, oclusão arterial assintomática, necessidade de reparo vascular cirúrgico e infecção local.

Por meio de hiperextensão do punho e infiltração de 1-2 ml de xilocaína a $2 \%$, puncionava-se a artéria radial $1 \mathrm{~cm}$ proximal ao processo estiloide do rádio, utilizando-se agulha com cateter de polietileno tipo Jelco $^{\text {TM }}$ calibre 20 e técnica de Seldinger. Após a punção, introduzia-se um fio-guia de 0,021 polegada, seguido de pequena incisão cutânea com lâmina de bisturi no 11 e inserção de introdutor curto $(<11 \mathrm{~cm})$ 5-7 F. Administrava-se uma solução contendo 5.000 UI de sulfato de heparina e $10 \mathrm{mg}$ de mononitrato de isossorbida através da extensão do introdutor. Ao término do procedimento, removia-se imediatamente o introdutor e obtinha-se hemostasia com curativo compressivo por meio de bandagem elástica adesiva porosa (Tensoplast ${ }^{\mathrm{TM}}$ ). Procedia-se a exame clínico do sítio de punção e avaliação do pulso radial no momento da alta, cerca de duas a três horas após a realização de procedimentos diagnósticos e na manhã seguinte à realização de procedimentos terapêuticos.

As variáveis categóricas foram expressas em frequência e porcentagem, comparadas com o teste de qui-quadrado. As variáveis contínuas foram expressas em média e desvio padrão, comparadas com o teste $t$ de Student ou teste exato de Fisher. Foram considerados estatisticamente significantes os resultados com valor de $\mathrm{P}<0,05$. Utilizou-se o programa SPSS, versão 12.0 (SPSS Inc., Chicago, Estados Unidos).

\section{RESULTADOS}

No período de maio de 2008 a setembro de 2009, 2.652 pacientes, dos quais 1.085 (40,9\%) pertencentes ao sexo feminino, submeteram-se a procedimentos coronários invasivos diagnósticos e terapêuticos. Destes, $988(91 \%)$ foram realizados através do acesso radial, compondo a amostra avaliada no registro, sendo os demais através dos acessos femoral (6\%) ou ulnar (3\%). As características demográficas basais dos pacientes estão expressas na Tabela 1. A média de idade foi de 62,5 anos, sendo $32,4 \%$ portadores de 


\section{Tabela 1 \\ Características demográficas e clínicas basais de mulheres submetidas a procedimentos coronários pelo acesso radial}

\begin{tabular}{lc}
\hline Variáveis & n = 988 \\
\hline Idade, anos & $62,5 \pm 11,4$ \\
Índice de massa corpórea, kg/m² & $22,8 \pm 0,3$ \\
Área de superfície corpórea, m² & $1,8 \pm 0,1$ \\
Hipertensão arterial sistêmica, n (\%) & $841(85,1)$ \\
Diabetes melito, n (\%) & $320(32,4)$ \\
Dislipidemia, n (\%) & $467(47,3)$ \\
Tabagismo, n (\%) & $202(20,4)$ \\
Histórico familiar de insuficiência & $188(19)$ \\
coronária, n (\%) & $95(9,6)$ \\
Infarto agudo do miocárdio prévio, n (\%) & $78(7,9)$ \\
Intervenção coronária percutânea & \\
prévia, n (\%) & $23(2,3)$ \\
Revascularização miocárdica prévia, n (\%) & $30(3)$ \\
Acidente vascular encefálico prévio, n (\%) & $541(54,8)$ \\
Angina estável/isquemia silenciosa, n (\%) & $392(39,7)$ \\
SCA sem supradesnível de ST, n (\%) & $55(5,5)$ \\
SCA com supradesnível de ST, n (\%) & \\
\hline
\end{tabular}

$\mathrm{n}$ = número de pacientes; SCA = síndrome coronária aguda.

diabetes melito, com índice de massa corpórea médio de $22,8 \mathrm{~kg} / \mathrm{m}^{2}$ e quadro de angina estável ou isquemia silenciosa como apresentação clínica preponderante $(54,8 \%)$.

O acesso radial direito foi utilizado em $95 \%$ dos casos. Coronariografia correspondeu a 828 (83,8\%) procedimentos, intervenção coronária percutânea eletiva a $84(8,5 \%)$, e intervenção coronária percutânea imediatamente após a coronariografia (ad hoc) a $76(7,7 \%)$. Sedação constituída por benzodiazepínico (diazepam/ midazolam) endovenoso isolado ou em associação com opioide (fentanil) foi empregada em $16 \%$ dos pacientes. As características dos procedimentos estão expressas na Tabela 2. A taxa de sucesso foi de 97,1\%, sendo as causas mais frequentes de necessidade de mudança da via de acesso a presença de oclusão arterial prévia (braquial, tronco braquiocefálico ou radial), variações anatômicas da artéria radial (looping rádioulnar, hipoplasia radial), ocorrência de vasoespasmo e apoio inadequado para realização de intervenção coronária percutânea. $\mathrm{O}$ acesso radial esquerdo foi a via alternativa escolhida em $61 \%$ dos casos de crossover.

Dentre as complicações observadas com a utilização do acesso radial, a mais comum foi a oclusão arterial assintomática, sem repercussão isquêmica da mão, em 2,8\% dos casos (Tabela 3). Espasmo grave ocorreu em $0,8 \%$ dos procedimentos e hematomas ocorreram em $2,4 \%$, sendo mais de $90 \%$ restritos ao tecido subcutâneo e inferiores a $5 \mathrm{~cm}$ de extensão. Duas pacientes $(0,2 \%)$ apresentaram sangramento grave (uma hemorragia digestiva alta e uma queda de hemoglobina de $5 \mathrm{~g} / \mathrm{dl}$ ) com necessidade de transfusão sanguínea, nenhum deles relacionado à via de acesso. Houve um $(0,1 \%)$ episódio de acidente isquêmico transitório, com paresia de III par craniano, sem alteração radiológica à tomografia computadorizada de crânio e com resolução completa dos sintomas, um $(0,1 \%)$ infarto agudo do miocárdio periprocedimento, um $(0,1 \%)$ tamponamento cardíaco seguido de drenagem de Marfan em decorrência de perfuração de artéria circunflexa distal pelo fio-guia de 0,014 polegada, e um $(0,1 \%)$ episódio de dissecção de tronco de coronária esquerda durante coronariografia, com necessidade de cirurgia cardíaca de emergência e evoluindo a óbito.

Analisando-se pacientes do sexo feminino com $<65$ anos e com $\geq 65$ anos (Tabela 4), observou-se elevada taxa de sucesso do procedimento em ambos os grupos $(98,1 \%$ vs. 95,8\%; P = 0,65) e baixa ocorrência de eventos cardiovasculares adversos graves $(0,4 \%$ vs. $0,2 \% ; P>0,99)$. Na estratificação de acordo com o procedimento realizado, notou-se tendência a maior duração de fluoroscopia $(11,3 \pm 0,4$ minutos vs. 9,1 \pm $6,2$ minutos; $P=0,06)$, mas sem diferença em relação ao número de cateteres utilizados ou à duração total do procedimento, nas pacientes idosas submetidas a intervenção coronária percutânea. Quanto às demais pacientes, submetidas a procedimentos diagnósticos ou intervenções coronárias percutâneas ad hoc, não foram encontradas diferenças na comparação de quaisquer das características do procedimento ou dos eventos clínicos adversos entre os grupos com $<65$ anos e com $\geq 65$ anos.

\section{DISCUSSÃO}

Os achados do registro, envolvendo pacientes do sexo feminino, consecutivas, sem critérios de seleção, representativas da prática do mundo real, demonstraram que a utilização do acesso radial na realização de procedimentos coronários diagnósticos e terapêuticos, em um grupo sabidamente exposto a maior risco de complicações, é segura e eficaz. A obtenção da via de acesso e a conclusão do procedimento com sucesso, a despeito de aproximadamente $45 \%$ das pacientes apresentarem idade superior a 65 anos, foram elevadas $(97,1 \%)$, sem incrementos na duração total do procedimento ou de fluoroscopia, este último um fiel marcador de dificuldade e complexidade técnica. Também se atestou a segurança do acesso radial na redução de complicações vasculares relacionadas ao sítio de punção e de sangramentos graves, com taxa inferior a $0,5 \%$, apesar de $45 \%$ da amostra avaliada constituir-se de pacientes com diagnóstico de síndrome coronária aguda, sob regime antitrombótico agressi$v^{10}$. Pristipino et al., em coorte prospectiva semelhan- 
Tabela 2

Características dos procedimentos coronários realizados em mulheres pelo acesso radial, de acordo com a intervenção realizada

\begin{tabular}{lccc}
\hline Variáveis & CATE $(\mathbf{n}=\mathbf{8 2 8})$ & ICP $(\mathbf{n}=\mathbf{8 4})$ & ICP ad hoc $(\mathbf{n}=\mathbf{7 6})$ \\
\hline Sucesso do procedimento, $\mathrm{n}(\%)$ & $808(97,5)$ & $79(94)$ & $72(96)$ \\
Duração do procedimento, min & $17,6 \pm 10,9$ & $34,7 \pm 22,2$ & $43,9 \pm 18,8$ \\
Duração de fluoroscopia, min & $4,1 \pm 4$ & $10,3 \pm 8,3$ & $10,4 \pm 5,3$ \\
Número de cateteres utilizados, média \pm DP & $2,1 \pm 0,5$ & $1,3 \pm 0,7$ & $2,6 \pm 1$ \\
Diâmetro do cateter, F (\%) & $5(90,5)$ & $5(9,6)$ & $5(13,3)$ \\
& $6(9,4)$ & $6(87,8)$ & $6(86,7)$ \\
\end{tabular}

CATE = coronariografia; DP = desvio padrão; ICP = intervenção coronária percutânea; $\mathrm{n}$ = número de pacientes.

Tabela 3

Taxa de complicações periprocedimento com a utilização do acesso radial em mulheres

\begin{tabular}{lc}
\hline Variáveis & $\mathbf{n}=\mathbf{9 8 8}$ \\
\hline Espasmo grave da artéria radial, n (\%) & $8(0,8)$ \\
Pseudoaneurisma, n (\%) & 0 \\
Oclusão arterial assintomática, n (\%) & $28(2,8)$ \\
Fístula arteriovenosa, n (\%) & 0 \\
Hematoma, n (\%) & $24(2,4)$ \\
Tipo I, n (\%) & $15(1,5)$ \\
Tipo II, n (\%) & $8(0,8)$ \\
Tipo III, n (\%) & $1(0,1)$ \\
Tipo IV, n (\%) & 0 \\
Tipo V, n (\%) & 0 \\
Sangramento grave, n (\%) & $2(0,2)$ \\
Transfusão sanguínea, n (\%) & $2(0,2)$ \\
Cirurgia cardíaca de urgência, n (\%) & $2(0,2)$ \\
Infarto agudo do miocárdio, n (\%) & $1(0,1)$ \\
Acidente isquêmico transitório, n (\%) & $1(0,1)$ \\
Acidente vascular encefálico, n (\%) & 0 \\
Morte, n (\%) & $1(0,1)$ \\
\hline $\mathrm{n}$ = número de pacientes. & \\
\hline
\end{tabular}

te, envolvendo 838 mulheres submetidas a 900 procedimentos coronários diagnósticos e terapêuticos, dos quais 299 (33\%) foram realizados pelo acesso radial, demonstraram taxa de sucesso de $92 \%$ e ausência de episódios de sangramento grave, contra 25 casos entre aquelas que utilizaram o acesso femoral $(P=0,0008)^{11}$.

Estudos observacionais recentes envolvendo grandes casuísticas identificaram preditores de eventos adversos entre pacientes submetidos a procedimentos coronários invasivos. No registro multinacional Registry on IntraVenous anticoagulation In the Elective and primary Real world of Angioplasty (RIVIERA), que in- cluiu 7.962 pacientes submetidos a intervenção coronária percutânea, o sexo feminino mostrou-se preditor independente de sangramento. O acesso radial, por sua vez, além de associar-se a menor risco de morte e infarto agudo do miocárdio, foi a única variável a promover menor índice de sangramento ${ }^{12}$. O estudo italiano Prevention of VTE after Acute Ischemic Stroke with LMWH Enoxaparin (PREVAIL), com 1.052 pacientes, demonstrou que o sexo feminino foi preditor independente para a ocorrência de desfechos combinados tanto primários (sangramento, acidente vascular encefálico e complicação vascular na via de acesso) quanto secundários (mortalidade hospitalar e infarto agudo do miocárdio). Nesse registro, a utilização do acesso radial associou-se de forma independente e significativa à redução de desfechos adversos, à custa de maior taxa de crossover quando comparado ao acesso femoral $(6,5 \%$ vs. $0,7 \% ; \mathrm{P}<0,0001)$. Entretanto, operadores habituados ao uso da técnica radial (> 50\% dos procedimentos) apresentaram baixo índice de insucesso $(4,6 \%$ vs. $24,5 \% ; \mathrm{P}<0,0001)$, achado similar ao de nossa casuística ${ }^{13}$.

Sabe-se que a ocorrência de fenômenos hemorrágicos promove maior risco de morbidade e mortalidade, sendo atualmente recomendada sua inclusão na avaliação de desfechos adversos após realização de intervenção coronária percutânea ${ }^{14}$. O registro retrospectivo canadense Mortality benefit Of Reduced Transfusion after percutaneous coronary intervention via the Arm or Leg (M.O.R.T.A.L), abrangendo 32.822 pacientes, demonstrou que, ao reduzir a taxa de sangramento e a necessidade de transfusão sanguínea, o acesso radial, de forma adicional, reduziu a mortalidade aos 30 dias [odds ratio (OR): 0,71; intervalo de confiança de 95\% (IC 95\%): 0,61-0,82; P < 0,001) e aos 12 meses de seguimento (OR: 0,83; IC 95\%: 0,71-0,98; $\mathrm{P}<0,001)^{15}$. Resultados semelhantes foram reportados no estudo ComPaRison of Early Invasive and Conservative Treatment in Patients With Non-STElevation Acute Coronary Syndromes (PRESTO-ACS) envolvendo 2.142 pacientes com diagnóstico de sín- 
Andrade PB, et al. Segurança e Eficácia do Acesso Radial na Realização de Procedimentos Coronários Diagnósticos e Terapêuticos em Mulheres. Rev Bras Cardiol Invas. 2009;17(4):457-62.

Tabela 4

Comparação das características do procedimento e eventos clínicos, de acordo com a intervenção realizada, em pacientes $<65$ anos vs. $\geq 65$ anos

\begin{tabular}{|c|c|c|c|c|c|c|}
\hline \multirow[b]{2}{*}{ Variáveis } & \multicolumn{3}{|c|}{$\begin{array}{c}\text { Grupo I (< } 65 \text { anos) } \\
n=534\end{array}$} & \multicolumn{3}{|c|}{$\begin{array}{c}\text { Grupo II ( } \geq 65 \text { anos) } \\
n=454\end{array}$} \\
\hline & $\begin{array}{c}\text { CATE } \\
n=471\end{array}$ & $\begin{array}{c}I C P \\
n=30\end{array}$ & $\begin{array}{l}\text { Ad hoc } \\
\mathrm{n}=33\end{array}$ & $\begin{array}{c}\text { CATE* } \\
\text { n }=357\end{array}$ & $\begin{array}{c}\mathrm{ICP}^{+} \\
\mathrm{n}=54\end{array}$ & $\begin{array}{c}\text { Ad hoc } \\
\mathrm{n}=43\end{array}$ \\
\hline Sucesso do procedimento, n (\%) & $461(97,9)$ & $30(100)$ & $33(100)$ & $346(96,9)$ & $49(90,7)$ & $40(93)$ \\
\hline Duração do procedimento, min & $17,4 \pm 12,1$ & $31,3 \pm 23,9$ & $40,6 \pm 10,6$ & $17,7 \pm 8,9$ & $36,5 \pm 22,0$ & $46,5 \pm 22,9$ \\
\hline Duração da fluoroscopia, min & $4 \pm 4,5$ & $9,1 \pm 6,2$ & $10,4 \pm 4$ & $4,2 \pm 3,3$ & $11,3 \pm 0,4$ & $10,3 \pm 6,3$ \\
\hline Número de cateteres utilizados, média \pm DP & $2,1 \pm 0,6$ & $1,4 \pm 0,7$ & $2,5 \pm 0,8$ & $2,1 \pm 0,6$ & $1,3 \pm 0,6$ & $2,6 \pm 1,2$ \\
\hline Eventos cardiovasculares adversos graves, $\mathrm{n}(\%)$ & o) $1(0,2)$ & $1(3,3)$ & 0 & 0 & $1(1,9)$ & 0 \\
\hline \multicolumn{7}{|c|}{$\begin{array}{l}\text { CATE = coronariografia; DP = desvio padrão; ICP = intervenção coronária percutânea; } n=\text { número de pacientes. } \\
\text { * CATE grupo I vs. grupo II (P > 0,10 para todas as comparações). } \\
+ \text { ICP grupo I vs. grupo II ( } \mathrm{P}=0,06 \text { para comparação de tempo de fluoroscopia; } P>0,10 \text { para demais comparações }) \text {. } \\
\text { ‡ ICP ad hoc grupo I vs. grupo II (P>0,10 para todas as comparacões). }\end{array}$} \\
\hline
\end{tabular}

drome coronária aguda sem supradesnível de ST submetidos a intervenção coronária percutânea ${ }^{16}$. A redução da taxa de sangramento proporcionada pelo acesso radial, quando comparado ao femoral (0,7\% vs. 2,4\%; $\mathrm{P}=0,05)$, associou-se a melhor prognóstico e sobrevida livre de morte e infarto agudo do miocárdio em um ano $(95,1 \%$ vs. $91,7 \% ; P=0,05)$.

Com base nesses dados, o emprego da técnica radial em mulheres torna-se recomendável. Apesar de exibirem menor incidência de doença aterosclerótica e de se submeterem a menor número de intervenções coronárias percutâneas, paradoxalmente as mulheres apresentam elevadas taxas de sangramento intra-hospitalar quando comparadas aos homens. Assim, a utilização da técnica radial ofereceria a perspectiva de equalização das taxas de complicações vasculares relacionadas à via de acesso. Em análise post-hoc do estudo EASY, em que 298 mulheres e 1.050 homens foram submetidos a intervenção coronária percutânea transradial em uso concomitante de ácido acetilsalicílico, clopidogrel e abciximab, embora as mulheres apresentassem maior prevalência de comorbidades, não houve diferença quanto à ocorrência de sangramento grave aos 30 dias entre os grupos $(2,4 \% \text { vs. } 1,1 \% ; P=0,16)^{17}$. Nunes et al., em estudo envolvendo 2.492 pacientes, sendo 942 do sexo feminino, submetidos a coronariografia ou intervenção coronária percutânea pela via radial, observaram maior incidência de hematomas locais de pequeno porte no grupo das mulheres (1,3\% vs. 0,4\%; $P=N S$ ), não sendo constatada, porém, diferença significativa entre os grupos quanto à ocorrência de hematomas de grande porte $(0,1 \% \text { vs. } 0,07 \% ; \mathrm{P}=\mathrm{NS})^{18}$.

Em se tratando de um registro observacional, o presente estudo possui como limitações sua natureza não-randômica, ser conduzido em um centro único e a ausência de seguimento clínico tardio.

\section{CONCLUSÃO}

O uso do acesso radial por operadores habituados à técnica na realização de procedimentos coronários diagnósticos e terapêuticos em mulheres, representativas da prática do mundo real, exibiu elevada taxa de sucesso, reduzido número de eventos cardiovasculares adversos e ocorrência virtualmente nula de sangramento grave relacionado ao sítio de punção.

\section{CONFLITO DE INTERESSES}

Os autores declararam inexistência de conflito de interesses.

\section{REFERÊNCIAS BIBLIOGRÁFICAS}

1. Singh M, Rihal CS, Gersh BJ, Lennon RJ, Prasad A, Sorajja $P$, et al. Twenty-five-year trends in in-hospital and long-term outcome after percutaneous coronary intervention: a singleinstitution experience. Circulation. 2007;115(22):2835-41.

2. Applegate RJ, Sacrinty MT, Kutcher MA, Kahl FR, Gandhi SK, Santos RM, et al. Trends in vascular complications after diagnostic cardiac catheterization and percutaneous coronary intervention via the femoral artery, 1998 to 2007. JACC Cardiovasc Interv. 2008;1(3):317-26.

3. Applegate RJ, Sacrinty MT, Kutcher MA, Baki TT, Gandhi SK, Kahl FR, et al. Vascular complications in women after catheterization and percutaneous coronary intervention 19982005. J Invasive Cardiol. 2007;19(9):369-74.

4. Brito Junior FS, Magalhães MA, Nascimento TCDC, Amorim IMG, Almeida BO, Abizaid A, et al. Incidência e preditores contemporâneos de complicações vasculares após intervenção coronária percutânea. Rev Bras Cardiol Invasiva. 2007;15(4): 394-9.

5. Doyle BJ, Rihal CS, Gastineau DA, Holmes DR Jr. Bleeding, blood transfusion, and increased mortality after percutaneous coronary intervention: implications for contemporary practice. J Am Coll Cardiol. 2009;53(22):2019-27.

6. Agostoni $\mathrm{P}$, Biondi-Zoccai GG, de Benedictis $\mathrm{ML}$, Rigattieri S, Turri M, Anselmi M, et al. Radial versus femoral approach 
for percutaneous coronary diagnostic and interventional procedures. Systematic overview and meta-analysis of randomized trials. J Am Coll Cardiol. 2004;44(2):349-56.

7. Jolly SS, Amlani S, Hamon M, Yusuf S, Mehta SR. Radial versus femoral access for coronary angiography or intervention and the impact on major bleeding and ischemic events: a systematic review and meta-analysis of randomized trials. Am Heart J. 2009;157(1):132-40.

8. Yoo BS, Yoon J, Ko JY, Kim JY, Lee SH, Hwang SO, et al. Anatomical consideration of the radial artery for transradial coronary procedures: arterial diameter, branching anomaly and vessel tortuosity. Int J Cardiol. 2005;101(3):421-7.

9. Bertrand OF, De Larochellière R, Rodés-Cabau J, Proulx G, Gleeton O, Nguyen CM, Déry JP, Barbeau G, Noel B, Larose E, Poirier P, Roy L; Early Discharge After Transradial Stenting of Coronary Arteries Study Investigators. A randomized study comparing same-day home discharge and abciximab bolus only to overnight hospitalization and abciximab bolus and infusion after transradial coronary stent implantation. Circulation. 2006;114(24):2636-43. Comment in: Circulation. 2006; 114(24):2578-80.

10. Tebet MA, Andrade PB, Gentile M, Mattos LA, Labrunie A. Angioplastia primária via radial com doses aumentadas de tirofiban: uma técnica segura e efetiva. Rev Bras Cardiol Invasiva. 2007;15(3):255-60.

11. Pristipino C, Pelliccia F, Granatelli A, Pasceri V, Roncella A, Speciale G, et al. Comparison of access-related bleeding complications in women versus men undergoing percutaneous coronary catheterization using the radial versus femoral artery. Am J Cardiol. 2007;99(9):1216-21.

12. Montalescot G, Öngen Z, Guindy R, Sousa A, Lu SZ, Pahlajani D, Pellois A, Vicaut E; for the RIVIERA Investigators. Predictors of outcome in patients undergoing $\mathrm{PCI}$. Results of the RIVIERA study. Int J Cardiol. 2008;129(3):379-87.
13. Pristipino C, Trani C, Nazzaro MS, Berni A, Patti G, Patrizi R, Pironi B, Mazzarotto P, Gioffré G, Biondi-Zoccai GG, Richichi G; Prospective Registry of Vascular Access in Interventions in Lazio Region Study Group. Major improvement of percutaneous cardiovascular procedure outcomes with radial artery catheterization: results from the PREVAIL study. Heart. 2009;95:4(6)76-82.

14. Ndrepepa G, Berger PB, Mehilli J, Seyfarth M, Neumann FJ, Schömig A, et al. Periprocedural bleeding and 1-year outcome after percutaneous coronary interventions: appropriateness of including bleeding as a component of a quadruple end point. J Am Coll Cardiol. 2008:51(7):690-7. Comment in: J Am Coll Cardiol. 2008;51(7):698-700.

15. Chase AJ, Fretz EB, Warburton WP, Klinke WP, Carere RG, $\mathrm{Pi} D$, et al. Association of the arterial access site at angioplasty with transfusion and mortality: the M.O.R.T.A.L study (Mortality benefit Of Reduced Transfusion after percutaneous coronary intervention via the Arm or Leg). Heart. 2008;94(8):1019-25. Comment in: Heart. 2008;94(12):1530-2.

16. Sciahbasi A, Pristipino C, Ambrosio G, Sperduti I, Scabbia EV, Greco C, et al. Arterial access-site-related outcomes of patients undergoing invasive coronary procedures for acute coronary syndromes (from the ComPaRison of Early Invasive and Conservative Treatment in Patients With Non-ST-ElevatiOn Acute Coronary Syndromes [PRESTO-ACS] Vascular Substudy). Am J Cardiol. 2009;103(6):796-800.

17. Tizón-Marcos H, Bertrand OF, Rodés-Cabau J, Larose E, Gaudreault V, Bagur R, et al. Impact of female gender and transradial coronary stenting with maximal antiplatelet therapy on bleeding and ischemic outcomes. Am Heart J. 2009;157(4): 740-5.

18. Nunes GL, Finger CS, Alves L, Costa AP, Nazareth C, Souza $\mathrm{E}$, et al. Aplicabilidade do acesso transradial em mulheres. Arq Bras Cardiol. 2002;79:III-61. [Apresentado no 57ํㅡㄹ Congresso Brasileiro de Cardiologia. 2002. São Paulo, 21-24 setembro]. 